\title{
Analysing $H(z)$ data using two-point diagnostics
}

\author{
Kyle Leaf $^{1 \star}$ and Fulvio Melia ${ }^{2 \star} \dagger$ \\ ${ }^{1}$ Department of Physics, The University of Arizona, AZ 85721, USA \\ ${ }^{2}$ Department of Physics, The Applied Math Program, and Department of Astronomy, The University of Arizona, AZ 85721 , USA
}

Accepted 2017 June 7. Received 2017 June 7; in original form 2017 April 20

\begin{abstract}
Measurements of the Hubble constant $H(z)$ are increasingly being used to test the expansion rate predicted by various cosmological models. But the recent application of two-point diagnostics, such as $\operatorname{Om}\left(z_{i}, z_{j}\right)$ and $\operatorname{Omh}^{2}\left(z_{i}, z_{j}\right)$, has produced considerable tension between $\Lambda$ CDM's predictions and several observations, with other models faring even worse. Part of this problem is attributable to the continued mixing of truly model-independent measurements using the cosmic-chronometer approach, and model-dependent data extracted from baryon acoustic oscillations. In this paper, we advance the use of two-point diagnostics beyond their current status, and introduce new variations, which we call $\Delta h\left(z_{i}, z_{j}\right)$, that are more useful for model comparisons. But we restrict our analysis exclusively to cosmic-chronometer data, which are truly model independent. Even for these measurements, however, we confirm the conclusions drawn by earlier workers that the data have strongly non-Gaussian uncertainties, requiring the use of both 'median' and 'mean' statistical approaches. Our results reveal that previous analyses using two-point diagnostics greatly underestimated the errors, thereby misinterpreting the level of tension between theoretical predictions and $H(z)$ data. Instead, we demonstrate that as of today, only Einstein-de Sitter is ruled out by the two-point diagnostics at a level of significance exceeding $\sim 3 \sigma$. The $R_{\mathrm{h}}=c t$ universe is slightly favoured over the remaining models, including Lambda cold dark matter and Chevalier-Polarski-Linder, though all of them (other than Einstein-de Sitter) are consistent to within $1 \sigma$ with the measured mean of the $\Delta h\left(z_{i}, z_{j}\right)$ diagnostics.
\end{abstract}

Key words: galaxies: distances and redshifts-galaxies: evolution-large-scale structure of Universe - cosmology: observations - cosmology: theory.

\section{INTRODUCTION}

Lambda cold dark matter $(\Lambda \mathrm{CDM})$ has done reasonably well accounting for a broad range of data and is therefore correctly viewed as the current standard model of cosmology (see, e.g. Planck Collaboration XXIII 2014). But recent analyses of the two-point correlation function of the cosmic microwave background (Melia 2014; Copi et al. 2015), as well as the $\operatorname{Om}\left(z_{i}, z_{j}\right)$ and $\operatorname{Omh}^{2}\left(z_{i}, z_{j}\right)$ diagnostics applied to measurements of the Hubble expansion rate $H(z)$ (Shafieloo, Sahni \& Starobinsky 2012; Sahni, Shafieloo \& Starobinsky 2014), appear to have revealed significant tension between its predictions and recent measurements (see, e.g. Zheng et al. 2016).

In this paper, we directly address the problems highlighted by the various analyses carried out with the $H(z)$ data, which apparently do not confirm the anticipated transition from early deceleration

\footnotetext{
^E-mail: kyleaf@email.arizona.edu (KL); melia@physics.arizona.edu (FM)

$\dagger$ John Woodruff Simpson Fellow.
}

to more recent acceleration in the cosmic expansion rate (Jimenez \& Loeb 2002; Moresco et al. 2016a). With this type of work, one typically compiles a unified sample of $H(z)$ versus redshift measurements based on various approaches, including the determination of differential ages using cosmic chronometers (Moresco et al. 2016b) and the inference of a characteristic distance scale revealed in baryon acoustic oscillations (BAOs; see, e.g. Blake et al. 2012).

However, it is inadvisable to make combined use of BAO measurements with other data. The BAO approach results in certain systematic effects that are difficult to quantify, certainly without the pre-assumption of a particular model. A BAO determination of $H(z)$ relies on both determining the actual baryon acoustic peak of a cluster, and the degree of contamination from the Alcock-Paczýnski effect (see, e.g. Melia \& López-Corredoira 2017).

To disentangle these effects, one must choose a cosmological model in order to determine the overall comoving acoustic scale, rendering the determination of $H(z)$ using $\mathrm{BAO}$ data unique to each model. BAO therefore provide useful data for analysing a specific model, but a comparative analysis of multiple cosmologies requires a careful recalibration of the acoustic scale for each individual 
case. In contrast, the differential age method is fundamentally much simpler. It has some systematic error based on using the Extended Press-Schechter approximation (Moresco et al. 2016a), but is heavily based on directly observable galaxy properties. Zheng et al. (2016) demonstrated via the analysis of several cosmological models that the merger of differential-age and BAO data resulted in statistically significant discrepancies when compared to the use of either data set on its own.

In this paper, we therefore restrict our attention to the sample of thirty model-independent cosmic chronometer measurements of $H(z)$ (Jimenez et al. 2003; Simon, Verde \& Jimenez 2005; Stern et al. 2010; Moresco et al. 2012; Zhang et al. 2014; Moresco 2015; Moresco et al. 2016a) to carry out a comparative test of several cosmological models, using variants of the previously introduced two-point correlation functions that may be applied to any cosmology, not solely those framed in the context of $\Lambda \mathrm{CDM}$. The previously defined $\operatorname{Om}\left(z_{i}, z_{j}\right)$ and $\operatorname{Omh}^{2}\left(z_{i}, z_{j}\right)$ diagnostics are based on the presence of an $\Omega_{\mathrm{m}}$ parameter in the formulation of the expansion rate $H(z)$, which restricts their use primarily to $\Lambda \mathrm{CDM}$ and its variations (Sahni et al. 2014). Throughout this paper, we define $\Omega_{\mathrm{m}} \equiv \rho_{\mathrm{m}} / \rho_{c}$ to be the matter energy density $\rho_{\mathrm{m}}$ today as a fraction of the critical density $\rho_{c} \equiv 3 c^{2} H_{0}^{2} / 8 \pi G$.

We will be comparing the observations with the predicted expansion rate in five models, including $\Lambda \mathrm{CDM}, w \mathrm{CDM}, R_{\mathrm{h}}=c t$ (Melia 2007; Melia \& Abdelqader 2009; Melia \& Shevchuk 2012; Melia 2016a, 2017), Chevalier-Polarski-Linder (Chevalier \& Polarski 2001; Linder 2003) and Einstein-de Sitter. Each of these cosmologies has a distinct relationship between $H(z)$ and $z$, and some have a parametrization that allows a best-fitting model to be identified via the optimization of certain free parameters. In previous studies, such a parametric fitting of the Hubble constant has been used with the $H(z)$ data to identify the ideal model parameters in $\Lambda \mathrm{CDM}$, and, in a few cases, a direct comparison has also been made between $\Lambda \mathrm{CDM}$ and $R_{\mathrm{h}}=c t$, though the latter has no free parameters for this purpose, so one must necessarily rely on information criteria to calculate relative likelihoods (Melia \& Maier 2013; Melia \& McClintock 2015; Wei, Melia \& Wu 2017).

The two-point diagnostics differ from this parametric fitting approach in several distinct ways, extending the model comparison in new and statistically meaningful directions. Previously, Ding et al. (2015) and Zheng et al. (2016) employed two two-point diagnostics (i.e. $O m\left[z_{i}, z_{j}\right]$ and $O m h^{2}\left[z_{i}, z_{j}\right]$ ), involving $\Omega_{\mathrm{m}}$ and $\Omega_{\mathrm{m}}^{2}$, applicable to several cosmological models. In these analyses, the two-point diagnostic was calculated based on the $\Omega_{\mathrm{m}}$ parameter in $\Lambda \mathrm{CDM}$, such that it should be consistent with zero if $\Lambda \mathrm{CDM}$ were the correct model. Then, the expectation value of that diagnostic was determined for all the models being considered, and the one most consistent with it was judged to be favoured over the others. Their results, however, were rather inconclusive. Zheng et al. (2016) found that, while $\Lambda \mathrm{CDM}$ was the model most favoured by the data among the tested sample, it was still nonetheless significantly inconsistent with the measurements, especially on the basis of 'median statistics' (see, e.g. Gott et al. 2001). Other dark-matter parameterizations, such as $w \mathrm{CDM}$ and CPL (Chevalier \& Polarski 2001; Linder 2003) performed even worse.

But one must be aware of the fact that in their diagnostic, a value of $H_{0}$ must be chosen a priori, even though there is still no consensus on what its true value is, given that high-redshift measurements (e.g. Planck Collaboration XXIII 2014) are in tension with $H_{0}$ measured locally (e.g. using Type Ia SNe; see Wei et al 2017, and references cited therein). In addition, we shall demonstrate in this paper that the statistics of two-point diagnostics has been used improperly in earlier applications, including those of Ding et al. (2015) and Zheng et al. (2016). Specifically, we shall show that previous applications of two-point diagnostics have overestimated the confidence level of their results, thereby overstating the tension between, for example, the measured $H(z)$ values and predictions by $\Lambda \mathrm{CDM}$. We will introduce new two-point diagnostics similar in spirit to the previously defined $\operatorname{Om}\left(z_{i}, z_{j}\right)$ and $\operatorname{Omh}^{2}\left(z_{i}, z_{j}\right)$, which, however, are appropriate for a broader range of models, including $R_{\mathrm{h}}=c t$, that was not included in Zheng et al.'s (2016) study. In spite of these improvements to the analysis of two-point diagnostics, however, we will confirm the results first demonstrated by Ding et al. (2015) and Zheng et al. (2016), that the errors reported in conjunction with the cosmic chronometer data are at least partially non-Gaussian.

Our goals in this paper are threefold. First, we extend the previous work with two-point diagnostics to a more comprehensive comparison of various models. As noted, our approach allows the inclusion of cosmologies whose parametrization is not based on $\Omega_{\mathrm{m}}$. Secondly, we restrict our analysis to the 30 cosmic chronometer measurements to ensure that the data are as free as possible of model biases. Thirdly, we present a more in-depth analysis of the statistics used with two-point diagnostics and demonstrate that one must modify the weighted-mean and median statistics when using quantities such as $\operatorname{Om}\left(z_{i}, z_{j}\right)$ and $\operatorname{Omh}^{2}\left(z_{i}, z_{j}\right)$. In Section 2, we reintroduce these previously defined diagnostics, and then define a similar diagnostic that is applicable to a broader range of models. We summarize the data used in this paper in Section 3, and then carry out the model comparisons using the new two-point diagnostic in Section 4. We end with our conclusions in Section 5.

\section{TWO-POINT DIAGNOSTICS}

Two-point diagnostics are statistical tools that allow one to comparatively analyse measurements in a pairwise fashion. Specifically, they allow one to employ $n$ measurements of a particular variable to construct $n(n-1) / 2$ comparisons between each pair of data. With the 30 cosmic chronometer determinations of $H(z)$ included in this study, we therefore have 435 comparisons. With this method, one can test both how well each pair of points fits a given cosmological model, and also determine how closely the stated error bars actually fit a normal distribution. Previous work by Zheng et al. (2016) concluded that the stated errors in the cosmic-chronometer data are strongly non-Gaussian, based on the application of the $\operatorname{Om}\left(z_{i}\right.$, $\left.z_{j}\right)$ and $\operatorname{Omh}^{2}\left(z_{i}, z_{j}\right)$ diagnostics. The method employed by Zheng et al., however, was based on the definition of a single diagnostic (for which the expectation value is zero in $\Lambda \mathrm{CDM}$ ), then to compare that single diagnostic with its expectation value for each model being tested. In this work, we have devized separate, but completely analogous diagnostics for each cosmology.

The apparent non-Gaussianity of the data motivates the use of a second approach, pioneered by Gott et al. (2001), based on 'median statistics', which does not rely on error propagation. In Gott et al.'s (2001) method, one takes advantage of the fact that for any single measurement taken from some truly random distribution function, one has a 50 per cent chance of that measurement being above the true median of the underlying distribution, with no assumption concerning its form. Therefore, if $N$ measurements are taken and ranked by their value, the probability that the true median lies between measurements $i$ and $i+1$ is given by the binomial distribution, such that

$P_{i}=\frac{2^{-N} N !}{i !(N-i) !}$ 
Table 1. Parameter values adopted from the joint analysis of Betoule et al. (2014), including Planck+WP+BAO+JLA.

\begin{tabular}{lcccc}
\hline Model & $\Omega_{\mathrm{m}}$ & $w_{\mathrm{de}}$ & $w_{0}$ & $w_{a}$ \\
\hline$R_{\mathrm{h}}=c t$ & - & - & - & - \\
$\Lambda \mathrm{CDM}$ & $0.305 \pm 0.010$ & - & - & - \\
$w \mathrm{CDM}$ & $0.303 \pm 0.012$ & $-1.027 \pm 0.055$ & - & - \\
$\mathrm{CPL}$ & $0.304 \pm 0.012$ & - & $-0.957 \pm 0.124$ & $-0.336 \pm 0.552$ \\
Einstein-de Sitter & 1.0 & - & - & - \\
\hline
\end{tabular}

Therefore, one finds the number of measurements away from the median one needs to take in order to find the 68 percent confidence region of the median. We shall demonstrate, however, that this approach is not strictly valid for two-point diagnostics, prompting the use of Monte Carlo simulations with mock data in order to determine the correct number of steps one ought to take away from the median to compute the actual confidence range. As detailed below, the expectation value for the two-point diagnostic introduced here is zero for the true cosmology. Based on (the modified) median statistics, the confidence with which each cosmology's median is consistent with zero will therefore determine which cosmological model is favoured by the data.

Zheng et al. (2016) found for the $\operatorname{Om}\left(z_{i}, z_{j}\right)$ diagnostic that each cosmology they tested, i.e. $\Lambda$ CDM, wCDM and CPL, had a median inconsistent with zero at more than a 68 per cent confidence level. But we will show that this blanket negative outcome is due to an improper use of median statistics. Furthermore, while useful, the $\operatorname{Om}\left(z_{i}, z_{j}\right)$ diagnostic is suitable only for a cosmology with an $\Omega_{\mathrm{m}}$ term. Some cosmologies, such as $R_{\mathrm{h}}=c t$ and EdS, lack a parameter analogous to $\Omega_{\mathrm{m}}$, so the $\operatorname{Om}\left(z_{i}, z_{j}\right)$ and $\operatorname{Omh}^{2}\left(z_{i}, z_{j}\right)$ diagnostics cannot be used, in general, for model selection. The $R_{\mathrm{h}}=c t$ model and Einstein-de Sitter have only one independent parameter, the Hubble constant $H_{0}$ today. Therefore, we will employ a comparable two-point diagnostic, valid for each cosmology, defined as

$\Delta h\left(z_{i}, z_{j}\right) \equiv \frac{1}{H_{0}}\left(\frac{H\left(z_{i}\right)}{E\left(z_{i}\right)}-\frac{H\left(z_{j}\right)}{E\left(z_{j}\right)}\right)$,

where the function $E(z)$ is cosmology-dependent. We stress that the actual value of $H_{0}$ does not affect the statistical comparison between the various models, and is used solely to provide a uniform scale for the diagnostic $\Delta h\left(z_{i}, z_{j}\right)$. As such, the measured variance of the Hubble constant is also irrelevant to the statistical outcome of the $\Delta h\left(z_{i}, z_{j}\right)$ analysis. So the Hubble constant may be chosen arbitrarily without affecting the confidence of the final result, and it is not necessary to use individually optimized values in each case. For simplicity, we use the value $H_{0}=70 \mathrm{~km} \mathrm{~s}^{-1} \mathrm{Mpc}^{-1}$ in all the figures and reported distributions. In each case, the $\Delta h\left(z_{i}, z_{j}\right)$ diagnostic should be zero if the cosmology being tested is a good match to the data. The models we will compare are as follows:

(i) The $R_{\mathrm{h}}=c t$ universe (a Friedmann-Robertson-Walker cosmology with zero active mass; Melia 2016a, 2017). This model is based on the total equation of state $\rho+3 p=0$, where $\rho$ and $p$ are the total energy density and pressure of the cosmic fluid (Melia 2007; Melia \& Abdelqader 2009; Melia \& Shevchuk 2012). In this cosmology, there are no free parameters once the Hubble constant $H_{0}$ is used to scale $\Delta h\left(z_{i}, z_{j}\right)$ :

$E^{R_{\mathrm{h}}=c t}(z)=(1+z)$.

(ii) Flat $\Lambda \mathrm{CDM}$ model, with matter and dark-energy densities fixed by the condition $\Omega_{\Lambda}=1-\Omega_{\mathrm{m}}$ (when radiation is insignificant). In addition to $H_{0}$, which is used to scale $\Delta h\left(z_{i}, z_{j}\right)$, this model has the parameter $\Omega_{\mathrm{m}}$, for which we use the prior value listed in Table 1. For this model,

$E^{\Lambda \mathrm{CDM}}(z)=\left[\Omega_{\mathrm{m}}(1+z)^{3}+\Omega_{\Lambda}\right]^{1 / 2}$.

(iii) Flat $w \mathrm{CDM}$ model, with matter and dark-energy densities fixed by the condition $\Omega_{\mathrm{de}}=1-\Omega_{\mathrm{m}}$ (again, when radiation is insignificant). In this case, the dark-energy equation of state, $w_{\mathrm{de}} \equiv$ $p_{\mathrm{de}} / \rho_{\mathrm{de}}$, in terms of the dark-energy pressure $p_{\mathrm{de}}$, is unconstrained. This model has two parameters, $\Omega_{\mathrm{m}}$ and $w_{\mathrm{de}}$, for which we use the prior values listed in Table 1. Here,

$E^{w \mathrm{CDM}}(z)=\left[\Omega_{\mathrm{m}}(1+z)^{3}+\Omega_{\mathrm{de}}(1+z)^{3\left(1+w_{\mathrm{de}}\right)}\right]^{1 / 2}$.

(iv) The Chevalier-Polarski-Linder model (Chevalier \& Polarski 2001; Linder 2003), with

$$
\begin{aligned}
E^{\mathrm{CPL}}(z)= & {\left[\Omega_{\mathrm{m}}(1+z)^{3}+\left(1-\Omega_{\mathrm{m}}\right)(1+z)^{3\left(1+w_{o}+w_{a}\right)}\right.} \\
& \left.\times \exp \left(-3 w_{a} z /[1+z]\right)\right]^{1 / 2} .
\end{aligned}
$$

Again, we use the prior values for the parameters indicated in Table 1.

(v) Einstein-de Sitter space, which contains only one matter. This model has no additional parameters once $H_{0}$ is used to scale $\Delta h\left(z_{i}, z_{j}\right)$. In this case,

$E^{\mathrm{EdS}}(z)=(1+z)^{3 / 2}$.

\section{OBSERVATIONS}

Parametrized cosmological models, such as those considered in this work, can be optimized to fit $H(z)$ measurements, of which there exist several types. In addition to optimizing the parameters of the standard model, they have been used to comparatively analyse the predictions of $\Lambda \mathrm{CDM}$ with those of other cosmologies. However, among these $H(z)$ measurements exist those which are model dependent. If a measurement of $H(z)$ is model dependent, it cannot be used to adequately compare multiple models. As of today, the cosmic chronometer approach is the only model-independent method of determining $H(z)$, and is therefore the method used exclusively in this work.

The cosmic chronometer approach used to determine the data used here constitutes a differential age method. By comparing the redshift-time derivative $(\mathrm{d} z / \mathrm{d} t)$ of galaxies undergoing passive evolution (that is, evolution on time-scales much longer than the age difference between the galaxies), one can infer the expansion rate $H(z)$ as a function of redshift. The galaxies used are elliptical and highly massive $\left(\gtrsim 10^{11} \mathrm{M}_{\odot}\right)$. They formed most ( $~ 90$ per cent) of their stellar mass in the redshift range of $2<z<3$, over a short time-frame of $\sim 0.1-0.3$ Gyr. At any given redshift, objects in this morphological class are thus the oldest objects in the Universe (Treu et al. 2005), and due to having nearly identical evolution history, 
Table 2. Hubble Parameter $H(z)$ measured at 30 different redshifts.

\begin{tabular}{|c|c|c|c|}
\hline Redshift & $\begin{array}{c}H(z) \\
\left(\mathrm{km} \mathrm{s}^{-1} \mathrm{Mpc}^{-1}\right)\end{array}$ & $\begin{array}{c}\sigma_{\mathrm{H}} \\
\left(\mathrm{km} \mathrm{s}^{-1} \mathrm{Mpc}^{-1}\right)\end{array}$ & Reference \\
\hline 0.07 & 69 & 19.6 & Zhang et al. (2014) \\
\hline 0.09 & 69 & 12 & Jimenez et al. (2003) \\
\hline 0.12 & 68.6 & 26.2 & Zhang et al. (2014) \\
\hline 0.17 & 83 & 8 & Simon et al. (2005) \\
\hline 0.1791 & 75 & 5 & Moresco et al. (2012) \\
\hline 0.1993 & 75 & 5 & Moresco et al. (2012) \\
\hline 0.2 & 72.9 & 29.6 & Zhang et al. (2014) \\
\hline 0.27 & 77 & 14 & Simon et al. (2005) \\
\hline 0.28 & 88.8 & 36.6 & Zhang et al. (2014) \\
\hline 0.3519 & 83 & 14 & Moresco et al. (2012) \\
\hline 0.3802 & 83 & 13.5 & Moresco et al. (2012) \\
\hline 0.4 & 95 & 17 & Simon et al. (2005) \\
\hline 0.4004 & 77 & 10.2 & Moresco et al. (2016a) \\
\hline 0.4247 & 87.1 & 11.2 & Moresco et al. (2016a) \\
\hline 0.4497 & 92.8 & 12.9 & Moresco et al. (2016a) \\
\hline 0.4783 & 80.9 & 9 & Moresco et al. (2016a) \\
\hline 0.48 & 97 & 62 & Stern et al. (2010) \\
\hline 0.5929 & 104 & 13 & Moresco et al. (2012) \\
\hline 0.6797 & 92 & 8 & Moresco et al. (2012) \\
\hline 0.7812 & 105 & 12 & Moresco et al. (2012) \\
\hline 0.8754 & 125 & 17 & Moresco et al. (2012) \\
\hline 0.88 & 90 & 40 & Stern et al. (2010) \\
\hline 0.9 & 117 & 23 & Simon et al. (2005) \\
\hline 1.037 & 154 & 20 & Moresco et al. (2012) \\
\hline 1.3 & 168 & 17 & Simon et al. (2005) \\
\hline 1.363 & 160 & 33.6 & Moresco (2015) \\
\hline 1.43 & 177 & 18 & Simon et al. (2005) \\
\hline 1.53 & 140 & 14 & Simon et al. (2005) \\
\hline 1.75 & 202 & 40 & Simon et al. (2005) \\
\hline 1.965 & 186.5 & 50.4 & Moresco (2015) \\
\hline
\end{tabular}

the ages of stars found in them can be combined with the measured redshifts to determine $H(z)$. Specifically, these measurements are based on the observation of the $4000 \AA$ break in the measured galaxy spectra. Metal absorption causes a discontinuity in the spectral continuum, such that the amplitude of the break increases linearly with stellar metallicity and stellar age (Moresco et al. 2016a). If the metal abundance is known, the age difference $\Delta t$ between two nearby galaxies can be determined by comparing their $4000 \AA$ amplitudes. With $\Delta t$ and $\Delta z$ known, one can then find the expansion rate according to

$H(z)=-\frac{1}{(1+z)} \frac{\mathrm{d} z}{\mathrm{~d} t} \approx-\frac{1}{(1+z)} \frac{\Delta z}{\Delta t}$.

The data used in this study are listed in Table 2. These are the same as those employed by Zheng et al. (2016), with the exception that their BAO measurements are here omitted.

\section{MODEL COMPARISONS}

Fig. 1 shows the complete two-point diagnostic for all 435 pairs of data for $R_{h}=c t$. The analogous figures for the other models are very similar to this and are not shown. Note that $\Delta z=z_{i}-z_{j}$ is always positive, so that $z_{i}>z_{j}$ in each evaluation of $\Delta h\left(z_{i}, z_{j}\right)$. The error bars are calculated using standard error propagation, assuming the provided errors are consistent with a normal distribution. The variance of each $\Delta h\left(z_{i}, z_{j}\right)$ diagnostic for each model is thus given as

$$
\begin{aligned}
\sigma_{\Delta h_{i j}}^{2}= & \frac{\sigma_{i}^{2}}{H_{0}^{2}\left[E\left(z_{i}\right)\right]^{2}}+\sum_{x}\left[\frac{\mathrm{d}}{\mathrm{d} x}\left(\frac{H\left(z_{i}\right)}{H_{0}\left[E\left(z_{i}\right)\right]}\right) \sigma_{x}\right]^{2} \\
& +\frac{\sigma_{j}^{2}}{H_{0}^{2}\left[E\left(z_{j}\right)\right]^{2}}+\sum_{x}\left[\frac{\mathrm{d}}{\mathrm{d} x}\left(\frac{H\left(z_{j}\right)}{H_{0}\left[E\left(z_{i}\right)\right]}\right) \sigma_{x}\right]^{2},
\end{aligned}
$$

where $E\left(z_{i}\right)$ is the appropriate function for the chosen cosmology and the summation over $x$ refers to each fitted parameter, such as $\Omega_{\mathrm{m}}$ and $w$ in $w \mathrm{cdm}$. There are no such terms for $R_{\mathrm{h}}=$ ct or EdS. Each $\sigma_{x}$ value is reported in Table 1 and $H_{0}$ is taken to have the uniform value $70 \mathrm{~km} \mathrm{~s}^{-1} \mathrm{Mpc}^{-1}$ in all cases, as previously noted. The choice of $H_{0}$ is just for normalization purposes and does not affect the statistical outcome in any way. The unweighted distribution of $\Delta h\left(z_{i}, z_{j}\right)$ values is shown in Fig. 2. A careful inspection reveals some departures from a pure Gaussian shape, for example, with tails in Figs 2(b)-(d) and, as we shall quantify below, a greater central peaking than one expects for a normal distribution.

Following our discussion above, we study the two-point diagnostic using both weighted-mean and median statistics. The weighted mean formula for the $\Delta h\left(z_{i}, z_{j}\right)$ diagnostic is just

$\Delta h_{\mathrm{w} . \mathrm{m} .}=\frac{\sum_{i=1}^{n-1} \sum_{j=i+1}^{n} \Delta h\left(z_{i}, z_{j}\right) / \sigma_{\Delta h_{i j}}^{2}}{\sum_{i=1}^{n-1} \sum_{j=i+1}^{n} 1 / \sigma_{\Delta h_{i j}}^{2}}$,

and its variance would naively appear to follow as

$\sigma_{\Delta h_{\mathrm{w} . \mathrm{m} .}}^{2}=\left(\sum_{i=1}^{n-1} \Sigma_{j=i+1}^{n} 1 / \sigma_{\Delta h_{i j}}^{2}\right)^{-1}$

the approach used by Ding et al. (2015) and Zheng et al. (2016) in their analyses. This determination of the error is inaccurate, however, and significantly underestimates the true error in the mean of the two-point diagnostic. We therefore propose the following approach which, as we shall see, will by construction alleviate much of the tension in the results of the previous works.

To illustrate this point, let us suppose that a measured parameter has a mean $\mu$ and standard deviation $\sigma$. Now pull three random measurements from this distribution, each with its own reported error $\sigma_{i}$, at increasing redshifts $z_{1}, z_{2}$, and $z_{3}$. Then, if we construct the two-point diagnostics consistent with the method outlined by Ding et al. (2105) and Zheng et al. (2016), and also followed in this paper, we find three values of the diagnostic for which the lower redshift is subtracted from a higher one, that is, $z_{3}-z_{2}$, $z_{3}-z_{1}$ and $z_{2}-z_{1}$. If we proceed to calculate the unweighted mean of these three diagnostic values, we find the following: $\mu_{2 \text {-point }}=\left(2 z_{3}\right.$ $\left.-2 z_{1}\right) / 3$. Through normal error propagation, this quantity has a standard deviation $\sigma=\sqrt{4 \sigma_{3}^{2}+4 \sigma_{1}^{2}} / 3$, which is very different from the more naive standard deviation given in equation (11), and in fact does not even depend on the middle measurement at all. This happens because taking the mean of a two-point diagnostic incurs a heavy contribution from the highest and lowest redshifts, but far less from the middle redshifts in the sample, as they are sometimes added and sometimes subtracted when evaluating the two-point function. Equation (11) ignores this eventuality. Therefore, the true method for determining the standard deviation of the two-point diagnostic must involve a careful application of standard error propagation to the weighted mean formula.

The actual calculation of the error in the mean becomes more complex when using weighted data. The weighted mean is calculated as in equation (10), though one must pay attention to the multiplicative terms that affect each of the original $n$ data points. 


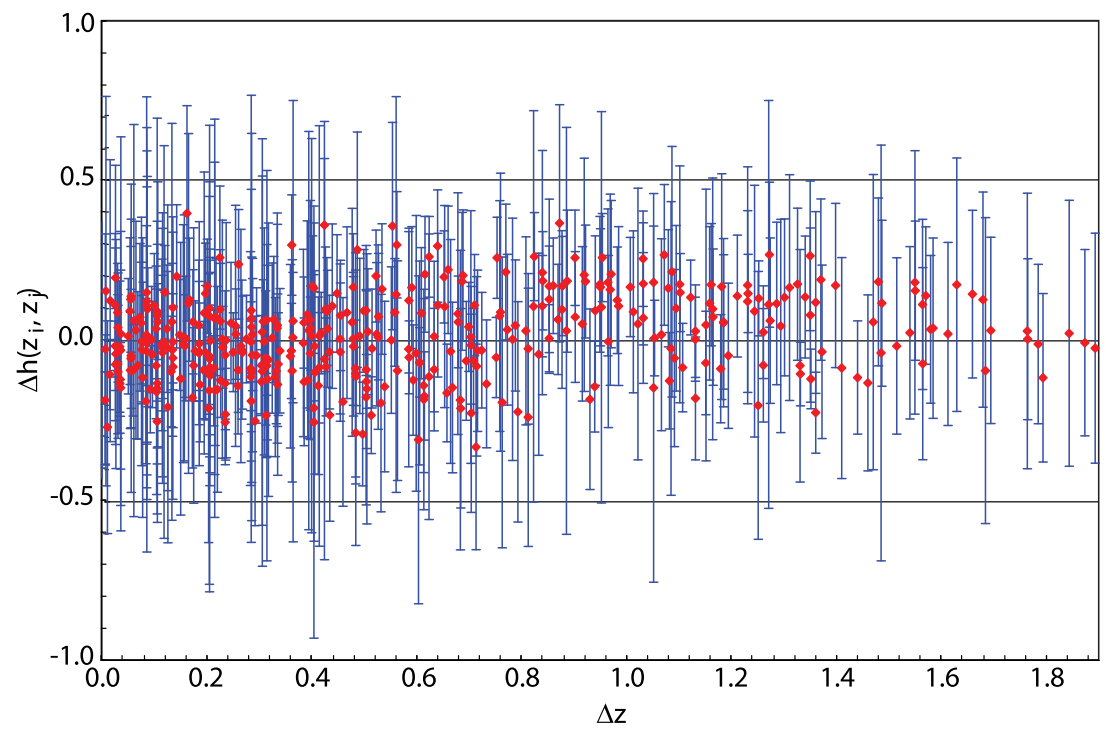

Figure 1. The $\Delta h\left(z_{i}, z_{j}\right)$ two-point diagnostic calculated for the sample of 30 cosmic-chronometer measurements of $H(z)$ in the $R_{\mathrm{h}}=c t$ cosmology. Red dots denote the calculated central values and the blue bars correspond to the uncertainties. If the cosmological model is correct, $\Delta h\left(z_{i}, z_{j}\right)$ should be zero for all values of $\Delta z$. The equivalent figures for the other cosmologies tested appear very similar, and are therefore omitted.

One finds that the weighted mean (equation 10) can be rearranged into the form

$\Delta h_{\mathrm{w} . \mathrm{m} .}=\frac{\sum_{i=1}^{n} \alpha_{i} H\left(z_{i}\right)}{\sum_{i=1}^{n-1} \Sigma_{j=i+1}^{n} 1 / \sigma_{\Delta h_{i j}}^{2}}$,

with

$\alpha_{i}=\Sigma_{j=1}^{i-1} \frac{1}{\sigma_{\Delta h_{i, j}}^{2}}-\Sigma_{k=1+i}^{N} \frac{1}{\sigma_{\Delta h_{i, j}}^{2}}$,

in which each $\alpha$ is the sum of every term in the numerator of the weighted mean that multiplied $H\left(z_{i}\right)$. Note that as with the constantdispersion case noted above, this tends to weight the measurements of $H(z)$ preferentially at the high and low redshifts, while underrepresenting the intermediate values. The true variance of the mean is therefore

$\Delta \sigma_{\text {w.m. }}^{2}=\frac{\sum_{i=1}^{n} \alpha_{i}^{2} \sigma^{2}\left(z_{i}\right)}{\left(\sum_{i=1}^{n-1} \Sigma_{j=i+1}^{n} 1 / \sigma_{\Delta h_{i j}}^{2}\right)^{2}}$,

which turns out to be always greater than the variance naively expected from equation (11).

Unlike the weighted mean, which assumes that all errors are Gaussian, median statistics makes no a priori assumption concerning the underlying distribution of a measurement compared with its true value. The probability that a given observation is above the 'true' median (i.e. the median of a very large number of measurements) is simply calculated by the binomial distribution given in equation (1), where $N$ is the total number of measurements, and $n$ is the value's position in the distribution. The smallest value has $n=1$, while the largest is $n=N$. However, despite making no assumptions about the error distribution, Gott et al.'s (2001) method does assume that all measurements are completely uncorrelated, which cannot be the case in a two-point measurement, where each of the $N$ data points affects the rest of the $N-1$ two-point values. As such, if the binomial distribution predicts that the true median lies within a certain number of measurements of the median of the two-point distribution, the actual 68 per cent confidence region is significantly greater.
To demonstrate this, we have generated mock data sets consisting of 30 determinations of $H(z)$, using the measured $H(z)$ values in Table 2 with their reported $\sigma_{\mathrm{H}}$ standard deviations, sampling them assuming a normal distribution. These mock $H(z)$ measurements were then used to determine a mock two-point set of 435 diagnostics for each of the models. We produced 1 million such mock data samples, recorded their medians, and then determined the 68 per cent confidence region of the median. Finally, we checked to see how many steps one must take away from the median of each mock data set in order to reach that 68 per cent region. Following this approach, we have found that the two-point diagnostic for all the models has a 68 percent chance of lying between the 192nd and 244th measurement (when ranked by value). The 218th measurement is the reported median of each set. This is a much larger range than that simply estimated using equation (1), for the simple reason that the two-point diagnostics are correlated. The proper use of median statistics in such cases is therefore not to calculate the confidence region based on a pure binomial distribution but, rather, to mitigate the impact of correlations by using a Monte Carlo approach. In this paper, we report the error above and below the median of each two-point diagnostic as the difference between the 218th and 192nd/244th ranked measurements.

Based on the weighted mean approach, using the corrected error estimation in equation (14), we find that the $\Delta h_{\mathrm{w} . \mathrm{m} \text {. }}$ two-point diagnostic applied to $R_{\mathrm{h}}=c t, \Lambda \mathrm{CDM}, w \mathrm{CDM}$ and $\mathrm{CPL}$ is consistent with zero to within $1 \sigma$ in every case (see Table 3 ). Einstein-de Sitter, however, is ruled out at better than $\sim 5 \sigma$. Furthermore, the value of the weighted mean for EdS is negative, implying that measurements taken at greater redshift point to smaller $H_{0}$ values than observations at low redshifts. The outcomes listed in Table 1 favour $R_{\mathrm{h}}=c t$ slightly over the other cosmologies, though no strong preference can be inferred from the two-point statistic used here. These results therefore weakly confirm earlier conclusions that $R_{\mathrm{h}}=c t$ is strongly preferred over $\Lambda \mathrm{CDM}$ based on the use of information criteria to assess the quality of the fits to the $H(z)$ data (Melia \& Maier 2013; Melia \& McClintock 2015; Wei et al. 2017). More importantly, in contrast to earlier reports (e.g. Zheng et al. 2016), 

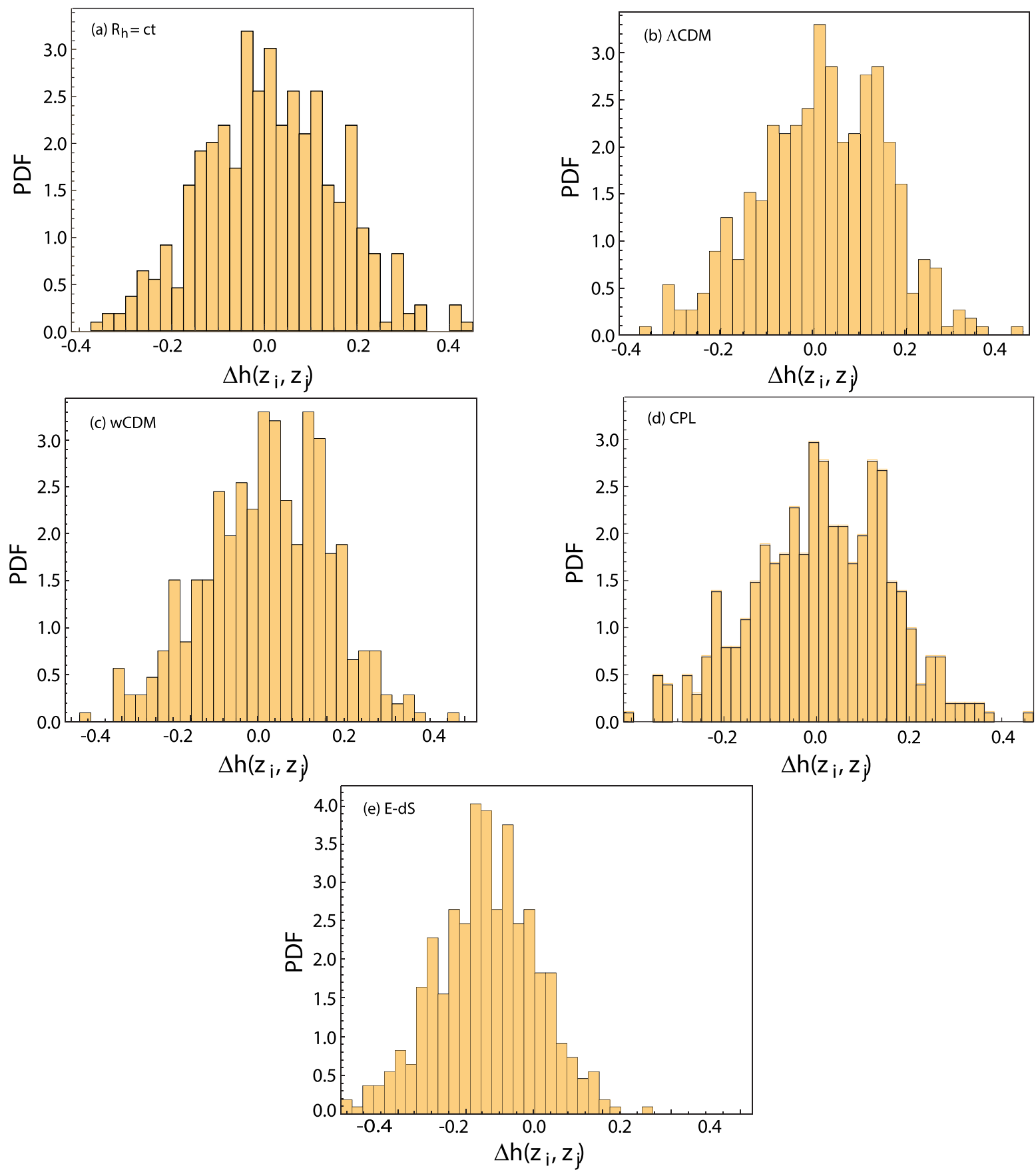

Figure 2. Histogram of the $\Delta h\left(z_{i}, z_{j}\right)$ two-point diagnostic calculated for the sample of 30 cosmic-chronometer measurements of $H(z)$, assuming (a) the $R_{\mathrm{h}}=c t$ cosmology; (b) $\Lambda \mathrm{CDM}$; (c) $w \mathrm{CDM}$; (d) CPL; and (e) Einstein-de Sitter.

Table 3. Statistical Outcomes.

\begin{tabular}{lrllrr}
\hline Model & \multicolumn{1}{c}{$\Delta h_{\text {w.m. }}$} & $\begin{array}{c}\text { Offset from } \\
\text { zero (w.m.) }\end{array}$ & $\left|N_{\sigma}\right|<1$ & $\Delta h_{\text {m.s. }}$ & $\begin{array}{c}68 \text { per cent range } \\
(\mathrm{m} . \mathrm{s} .)\end{array}$ \\
\hline$R_{\mathrm{h}}=c t$ & $0.00747 \pm 0.02786$ & $0.27 \sigma$ & 87.36 percent & 0.00879 & $(-0.01237,+0.02194)$ \\
$\Lambda \mathrm{CDM}$ & $0.01209 \pm 0.03082$ & $0.39 \sigma$ & 90.11 percent & 0.02126 & $(-0.01469,+0.02183)$ \\
$w \mathrm{CDM}$ & $0.01360 \pm 0.03122$ & $0.4355 \sigma$ & 90.34 percent & 0.02528 & $(-0.01780,+0.02118)$ \\
$\mathrm{CPL}$ & $0.01341 \pm 0.03942$ & $0.3402 \sigma$ & 97.47 percent & 0.02654 & $(-0.01580,+0.02362)$ \\
EdS & $-0.10851 \pm 0.02146$ & $5.06 \sigma$ & 85.06 percent & -0.10802 & $(-0.01756,+0.01248)$ \\
\hline
\end{tabular}


we find a much reduced tension between theoretical predictions and the actual measurements. As we have already noted, this difference is entirely due to our more careful handling of the weighted-mean statistics.

Note also that column 4 in Table 3 indicates a deviation from true Gaussian errors, confirming the pioneering analysis by Zheng et al. (2016) in this regard, who used the $\operatorname{Om}\left(z_{i}, z_{j}\right)$ diagnostic. For all the models we examine here, the percentage of pairs $N_{\sigma}$ with a $\Delta h\left(z_{i}, z_{j}\right)$ within $1 \sigma$ of the weighted mean is greater than the expected 68 percent if the errors associated with the measured $H(z)$ values (Table 2) are truly Gaussian. The fact that more measurements of $\Delta h\left(z_{i}, z_{j}\right)$ lie within $1 \sigma$ than predicted by Gaussianity (see also Fig. 2) suggests that the published error bars are generally larger than their actual values, or that there exists some generic systematic error that results in most measurements being offset from their true value in a similar manner.

One may already infer this for about half of the measurements in Table 2, for which both the statistical $\sigma_{\text {stat }}$ and systematic $\sigma_{\text {sys }}$ contributions to $\sigma_{i}$ have been reported (Gaztanaga, Cabré \& Hui 2009; Moresco et al. 2012, 2016a). In some cases, $\sigma_{\text {sys }} \sim \sigma_{\text {stat }}$, so even if only a portion of $\sigma_{\text {sys }}$ is correlated, the overall $\sigma_{i}$ calculated in quadrature overestimates the true Gaussian error. Unfortunately, this breakdown in statistical versus systematic errors is only known for a portion of the sample in Table 2. And even for those measurements where both $\sigma_{\text {stat }}$ and $\sigma_{\text {sys }}$ are available, one does not know which fraction of the latter is correlated. For the study reported in this paper, we have therefore settled on the simplest approach, which is to use all of the data in Table 2, with the caveat that the published errors are almost certainly overestimated. At least this approach ensures consistency across the entire sample, though the results do confirm that the errors are not perfectly Gaussian.

Of course, this also implies that the actual error in the weighted

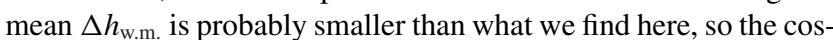
mologies we test are likely somewhat less consistent with $\Delta h_{\mathrm{w} . \mathrm{m} .}=0$ than is reported in Table 3. Clearly, this study will need to be updated once a better handle is available on the nature of $\sigma_{\text {sys }}$ for $H(z)$ measurements using cosmic chronometers.

Median statistics generally provides a picture consistent with this perspective. For Einstein-de Sitter, the result is similar to that of the weighted-mean approach, in that the true median in this model is entirely inconsistent with zero. The $R_{h}=c t$ universe is the only model for which a diagnostic value of zero lies within the 68 per cent confidence interval. All other models are inconsistent with zero to some degree, although $\Lambda \mathrm{CDM}$ is preferred over $w \mathrm{CDM}$ and CPL. Notice also the apparent existence of a double peak in the $\Lambda \mathrm{CDM}$, $w \mathrm{CDM}$ and CPL histograms (Fig. 2b-d). As noted earlier, the use of median statistics avoids the problem of the weighted mean, in not assuming how the data are randomly distributed. As such, results based on median statistics may be stronger than those derived from the weighted mean. Both reject EdS, however, and prefer $R_{h}=c t$ over other models compared here. Going forward, we suggest that our method of estimating the error in the median using Monte Carlo to construct mock samples is the proper way of finding the error associated with two-point diagnostics, rather than the binomial distribution used by Zheng et al. (2016) and others, given that the latter significantly underestimates the errors.

\section{CONCLUSION}

The previously introduced two-point diagnostics, such as $\operatorname{Om}\left(z_{i}, z_{j}\right)$, have been used successfully to test the viability of various cosmologies based on the measurement of $H(z)$. Here, we have extended this work by introducing a new two-point diagnostic, $\Delta h\left(z_{i}, z_{j}\right)$, which is more generally applicable to a wider range of models, including those whose formulation does not include the normalized matter density $\Omega_{\mathrm{m}}$. It allows for the analysis of $n(n-1) / 2$ pairs of data, offering different statistics than is available solely with methods that rely on just $n$ measurements.

This approach, however, also introduces some combinatorial effects that must be properly accounted for in order to draw accurate conclusions. The diagnostic we have introduced here allows for a fair test to determine whether the errors associated with the data are being estimated correctly. Earlier uses of $H(z)$ measurements included some model-dependent data, namely those based on BAO peaks in the galaxy distribution. But most of these measurements must preassume a cosmology in order to disentangle the BAO peak position from redshift-space distortions (RSD) due to internal galaxy motions. As such, BAO data tend to be incompatible with all models other than the one used to remove the RSD, and are therefore not useful when comparing different cosmologies for model selection, unless each model was carefully considered separately from the beginning, which has not been the case with existing data. In this paper, we have avoided all such biases, relying instead on cosmic chronometer observations, which tend to be independent of any model.

Using standard weighted-mean statistics, we have found that the $\Delta h\left(z_{i}, z_{j}\right)$ diagnostic is consistent with zero to within $1 \sigma$ for all the tested models, except for Einstein-de Sitter, which is ruled out at over $5 \sigma$. The caveat with this result is that our analysis has also demonstrated that the errors reported for $H(z)$ are not purely Gaussian. The true errors are almost certainly smaller than those published, or have a correlated systematic effect that would result in

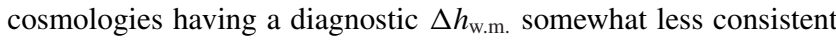
with zero than what we are reporting here.

Median statistics, based on the use of Monte Carlo methods to estimate the confidence region associated with the measured median, rather than simply using a binomial distribution that assumes uncorrelated data, has yielded a result consistent with that of the weighted mean. Einstein-de Sitter is again ruled out strongly, with $R_{h}=c t$ the significantly preferred model of those tested here. However, the confidence intervals of the median of these two-point diagnostics do not constitute a definitive rejection of any model other than EdS.

This result is intriguing, especially when contrasted with the analysis of the same data using the $\operatorname{Om}\left(z_{i}, z_{j}\right)$ diagnostic (Ding et al. 2015; Zheng et al. 2016). These earlier works used an improper statistical analysis, and did not include the $R_{\mathrm{h}}=c t$ universe in their comparisons, principally because the $\operatorname{Om}\left(z_{i}, z_{j}\right)$ diagnostic cannot be applied to it directly. Zheng et al. (2016) found that, while $\Lambda$ CDM is favoured over CPL and $w \mathrm{CDM}$, even $\Lambda \mathrm{CDM}$ itself has a two-point diagnostic that is strongly incompatible with zero. On the other hand, we have shown in this paper that two-point diagnostic errors reported in earlier work were severely underestimated.

The remaining issue is whether the results, we have derived here carry over to an analogous statistical analysis using the $\operatorname{Om}\left(z_{i}\right.$, $\left.z_{j}\right)$ and $\operatorname{Omh}^{2}\left(z_{i}, z_{j}\right)$ diagnostics. We have therefore repeated the work of Zheng et al. (2016) using these two-point diagnostics, both with their error methodology and our improved treatment that correctly accounts for correlations in the data. Given that $\Omega_{\mathrm{m}}$ is not a model parameter in all cosmologies, however, we have restricted this comparison to $\Lambda \mathrm{CDM}$ only. To summarize the results, we have found that, while the $\operatorname{Om}\left(z_{i}, z_{j}\right)$ diagnostic was inconsistent with its expected value of 0 at $2.8 \sigma$ based on median statistics and Zheng et al.'s incorrect error assessment, it is actually inconsistent with this value at only $0.96 \sigma$ when the errors are estimated correctly using our 
approach. With the use of weighted-mean statistics, this diagnostic was found by Zheng et al. to be inconsistent with 0 at $0.12 \sigma$, whereas the correct error handling yields an inconsistency at only the $0.04 \sigma$ level. The former comparison is more valid in this case, however, since we all agree that the reported errors are non-Gaussian. Using median statistics, Zheng et al. also found that the measured $\operatorname{Omh}^{2}\left(z_{i}\right.$, $z_{j}$ ) diagnostic is inconsistent with its expected value of 0.1426 at $4.4 \sigma$, while our corrected error assessment improves this to an inconsistency of $1.5 \sigma$.

The statistical analysis of the data using our $\Delta h\left(z_{i}, z_{j}\right)$ diagnostic therefore appears to be completely consistent with the results based on the use of $\operatorname{Om}\left(z_{i}, z_{j}\right)$ and $\operatorname{Omh}^{2}\left(z_{i}, z_{j}\right)$. The advantage of the former, however, is that it can be used for all cosmologies, not only those in which $\Omega_{\mathrm{m}}$ is a free parameter. Very importantly, we have confirmed that our improved error analysis significantly modifies the conclusions regarding which models are ruled out by the cosmic chronometer data, irrespective of which two-point diagnostic is used in the model comparisons. Based solely on these diagnostics, including $\Delta h\left(z_{i}, z_{j}\right)$, only Einstein-de Sitter is ruled out strongly by these observations, though $R_{\mathrm{h}}=c t$ is slightly preferred compared to the rest.

\section{ACKNOWLEDGEMENTS}

We are grateful to Rabindra Bhattacharya for assistance in finding the proper expression for the weighted mean of the two-point function. We are also grateful to the referee, Marek Biesiada, for his very thoughtful review, which has resulted in several notable improvements to the manuscript. FM is grateful to the Instituto de Astrofísica de Canarias in Tenerife and to Purple Mountain Observatory in Nanjing, China for their hospitality while part of this research was carried out. FM is also grateful for partial support to the Chinese Academy of Sciences Visiting Professorships for Senior International Scientists under grant 2012T1J0011, and to the Chinese State Administration of Foreign Experts Affairs under grant GDJ20120491013.

\section{REFERENCES}

Betoule M. et al., 2014. A\&A, 568, 22 Blake C. et al., 2012, MNRAS, 425, 405
Chevalier M., Polarski D., 2001, IJMP-D, 10, 213

Copi C., Huterer D., Schwarz D., Starkman G., 2015. MNRAS, 451, 2978

Ding X., Biesiada M., Cao S., Li Z., Zhu Z., 2015. ApJ, 803, L22

Gaztanaga E., Cabré A., Hui L., 2009. MNRAS, 399, 1663

Gott J. R., III, Vogeley M. S., Podariu S., Ratra B., 2001, Apj, 549, 1

Jimenez R., Loeb A., 2002. ApJ, 573, 37

Jimenez R., Verde L., Treu T., Stern D., 2003. ApJ, 593, 622

Linder E. V., 2003, PRL, 90, 091301

Melia F., 2007, MNRAS, 382, 1917

Melia F., 2014, A\&A, 561, A80

Melia F., 2016a, Front. Phys., 11, 119801

Melia F., 2017, Front. Phys., 12, 129802

Melia F., Abdelqader M., 2009, IJMP-D, 18, 1889

Melia F., López-Corredoira M., 2017, IJMP-D, 26, 1750055

Melia F., Maier R. S., 2013, MNRAS, 432, 2669

Melia F., McClintock T. M., 2015, AJ, 150, 119

Melia F., Shevchuk A. S. H., 2012, MNRAS, 419, 2579

Moresco M., 2015, MNRAS, 450, L16

Moresco M. et al., 2012, JCAP, 8, 006

Moresco M. et al., 2016a, JCAP, 05, 014

Moresco M., Jimenez R., Verde L., Cimatti A., Pozzetti L., Maraston C., Thomas D., 2016b, JCAP, 12, 039

Planck Collaboration XXIII, 2014, A\&A, 571, id.A23

Sahni V., Shafieloo A., Starobinsky A. A., 2014, ApJ, 793, L40

Shafieloo A., Sahni V., Starobinsky A. A., 2012, PhRvD, 86, 103527

Simon J., Verde L., Jimenez R., 2005, PRD, 71, 123001

Stern D., Jimenez R., Verde L., Stanford S. A., Kamionkowski M., 2010, ApJS, 188, 280

Treu T. et al., 2005. ApJ, 633, 174

Wei J.-J., Melia F., Wu X.-F., 2017. ApJ, 835, 270

Zhang C., Zhang H., Yuan S., Liu S., Zhang T.-J., Sun Y.-C., 2014. Res. Astron. Astrophys., 14, 1221

Zheng X., Ding X., Biesiada M., Cao S., Zhu Z.-H., 2016, ApJ, 825, 17

This paper has been typeset from a $\mathrm{T}_{\mathrm{E}} \mathrm{X} / \mathrm{LAT} \mathrm{T}$ file prepared by the author. 